\title{
BMJ Open Retrospective case review of missed opportunities for primary prevention of stroke and TIA in primary care: protocol paper
}

\section{Grace M Moran, Melanie Calvert, Max G Feltham, Tom Marshall}

To cite: Moran GM, Calvert M, Feltham MG, et al. Retrospective case review of missed opportunities for primary prevention of stroke and TIA in primary care: protocol paper. BMJ Open 2014;4:e006622. doi:10.1136/ bmjopen-2014-006622

- Prepublication history and additional material is available. To view please visit the journal (http://dx.doi.org/ 10.1136/bmjopen-2014006622).

Received 12 September 2014 Accepted 22 September 2014

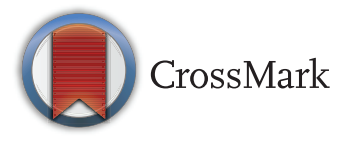

Primary Care Clinical Sciences, University of Birmingham, Birmingham, UK

Correspondence to Grace M Moran; gxt513@bham.ac.uk

\section{ABSTRACT}

Introduction: Stroke is a major health problem and transient ischaemic attack (TIA) is an important risk factor for stroke. Primary prevention of stroke and TIA will have the greatest impact on reducing the burden of these conditions. Evidence-based guidelines for stroke/TIA prevention identify individuals eligible for preventative interventions in primary care. This study will investigate: (1) the proportion of strokes/TIAs with prior missed opportunities for prevention in primary care; (2) the influence of patient characteristics on missed prevention opportunities and (3) how the proportion of missed prevention opportunities has changed over time.

Methods and analysis: A retrospective case review will identify first-ever stroke and patients with TIA between 2000 and 2013 using anonymised electronic medical records extracted from the health improvement network (THIN) database. Four categories of missed opportunities for stroke/TIA prevention will be sought: untreated high blood pressure in patients eligible for treatment (either blood pressure $\geq 160 / 100$ or $\geq 140 / 90 \mathrm{~mm} \mathrm{Hg}$ in patients at high cardiovascular disease (CVD) risk); patients with atrial fibrillation with high stroke risk and no anticoagulant therapy; no lipid modifying drug therapy prescribed in patients at high CVD risk or with familial hypercholesterolaemia. The proportion of patients with each missed opportunity and multiple missed opportunities will be calculated. Mixed effect logistic regression will model the relationship between demographic and patient characteristics and missed opportunities for care; practice will be included as a random effect.

Ethics and dissemination: THIN data collection was approved by the NHS South East Multi-centre Research Ethics Committee (MREC) in 2003. This study was approved by the independent scientific review committee in May 2013. Dissemination of findings has the potential to change practice, improve the quality of care provided to patients and ultimately reduce the incidence of strokes and TIAs. Findings will be published in a peer-reviewed journal and disseminated at national and international conferences.

\section{INTRODUCTION}

Stroke is one of the leading causes of mortality and disability in the UK. ${ }^{1}$ Transient ischaemic attack (TIA) is characterised by transient stroke-like symptoms and is an important risk factor for stroke. Given there are approximately 110000 first strokes and 46000 first TIAs a year reported in the $\mathrm{UK},{ }^{1}{ }^{2}$ primary prevention is important to reduce the burden of stroke and TIA. ${ }^{3}$

Understanding risk factors for stroke and TIA is important to identify people at high risk and implement preventative intervention. Hypertension is arguably the most welldocumented risk factor; a positive and continuous relationship has been shown between increasing blood pressure and stroke. ${ }^{4}$ Atrial fibrillation is associated with a fivefold increase in stroke risk. ${ }^{6}$ In addition, evidence suggests strokes in patients with atrial fibrillation are associated with greater disability and higher mortality rates. ${ }^{6}$ Cholesterol has been identified as risk factor for stroke; however, the relationship is not well characterised and is likely to be complex. Epidemiological studies have observed an association between lipid levels and stroke ${ }^{7}$ but findings are inconsistent across studies. ${ }^{8}$ On the other hand, a systematic review of 26 studies found a $20 \%$ reduction in strokes with statin therapy compared with placebo or usual care. ${ }^{9}$ Other conditions found to increase stroke risk include diabetes and cardiovascular disease (CVD).$^{10}$ In addition, lifestyle factors related to diet, obesity, physical inactivity, smoking and alcohol intake have been identified as risk factors for stroke and, moreover, have been shown to interact with other risk factors to exacerbate the risk. For example, obesity is associated with hypertension and high cholesterol. ${ }^{11}$

Age is an important risk factor; incidence and prevalence of stroke and TIA increases with age $^{2}$ and stroke risk doubles every decade over 55 years. ${ }^{12}$ Male sex has also been identified as a risk factor with men having a higher incidence of stroke compared with women. ${ }^{13}$ Although the mechanism is not fully understood, increased stroke 
incidence has been observed in south Asian and Afro-Caribbean ethnic groups. ${ }^{14}$

A person's stroke and CVD risk is determined by the combination of different risk factors. Multivariable CVD risk equations have been developed to identify high-risk patients and express risk as a probability over a period of time. ${ }^{15}$ Multiple risk equations exist, although they differ slightly in the risk factors included, the majority include age, sex, blood pressure, cholesterol, smoking and diabetes. ${ }^{16}$ Patients with atrial fibrillation stroke risk is increased by independent risk factors. ${ }^{17}$ Stroke risk algorithms for these patients include the risk factors: age, congestive heart failure, hypertension, diabetes and previous stroke or TIA. ${ }^{6}$

Primary care offers the best opportunity to identify people at high risk of stroke and TIA and administer preventative action. Studies have shown that pharmacological treatments reduce risk by a constant proportion. ${ }^{18}$ Evidence-based guidelines relevant to stroke prevention have been developed for hypertension, atrial fibrillation and lipid modification. Hypertension guidelines advise antihypertensive drug therapy is initiated in people with sustained blood pressure $\geq 160 / 100 \mathrm{~mm} \mathrm{Hg}$ or a lower threshold of $\geq 140 / 90 \mathrm{~mm} \mathrm{Hg}$ for people with established CVD, diabetes or an estimated CVD risk of $\geq 20 \%$ over 10 years. ${ }^{19}$ Atrial fibrillation guidelines recommend patients' stroke risk is assessed using an algorithm and high-risk patients should be prescribed anticoagulant therapy. ${ }^{6}$ Lipid modification guidelines advise lipid lowering drug therapy should be initiated in people considered high risk as opposed to measuring blood cholesterol levels. Guidelines regard high risk as people with established CVD, diabetes or an estimated CVD risk of $\geq 20 \%$ over 10 years and endorse prescription of statins. ${ }^{16}$

Despite the extensive evidence-based guidelines to reduce stroke risk, patients who present at hospital with first stroke have been found to have multiple untreated or undertreated risk factors. ${ }^{20}$ Furthermore, it has been found that some general practitioners (GPs) accept higher blood pressure thresholds than recommended by the guidelines ${ }^{21}$ and overestimate the proportion of their patients with controlled blood pressure. ${ }^{22}$ Existing studies of adherence to stroke prevention guidelines are limited as they use hypothetical questionnaires or retrospective interviews, where responses may differ from actual practice, and often focus on only one risk factor. Considering the complexity of the risk factors for firsttime stroke and TIA, a large-scale UK study using reallife primary care data to examine the administration of primary prevention is an important and necessary step to reduce the burden of strokes and TIAs on the National Health Service (NHS) and society.

\section{AIMS}

The study aims to investigate: (1) the proportion of first strokes and TIAs with prior missed opportunities for prevention in primary care; (2) the influence of patient characteristics on missed prevention opportunities and (3) how proportions of missed prevention opportunities have changed over time.

\section{METHODS AND ANALYSIS \\ Study design}

A retrospective case review of patients with a first-ever stroke or TIA.

\section{Data source}

Relevant data will be extracted from the health improvement network (THIN), a large database of anonymised UK electronic primary care records. Data are comprised of over 500 general practices, include 11.9 million patients and cover $6 \%$ of the UK population. ${ }^{23}$ The information recorded within THIN is comprehensive and includes demographics, diagnoses, prescriptions, additional health information (eg, lifestyle factors), socioeconomic data and free-text comments. Data are coded using drug codes which correspond to British National Formulary (BNF) chapters $^{24}$ and Read codes (V.2). ${ }^{25}$ THIN data collection was approved by the NHS South East Multi-centre Research Ethics Committee (MREC) in $2003 .{ }^{26}$

\section{Population}

Patients with stroke and TIA between 2000 and 2013 will be identified and relevant data extracted from the THIN database. This study will investigate primary prevention of stroke and TIA; therefore, will comprise of patients with first-ever stroke and TIA. However, as TIA is a risk factor for stroke, patients will be categorised into three groups: stroke only, TIA only, stroke with a history of TIA. To exclude childhood stroke, only patients with a diagnosis of stroke or TIA over 18 years will be included in the study. Date of stroke or TIA will be taken as the index date, and patients must be registered for at least 1 year prior to the index date to allow sufficient time for risk factor data to be recorded. To ensure data quality, the index date must occur at least 1 year after the practice had begun using Vision software, and after the practice date of acceptable mortality recording, the year mortality rates for the practice correspond to expected regional mortality rates. ${ }^{27}$

\section{Outcomes}

Four missed opportunities for primary stroke and TIA prevention have been defined through consulting relevant guidelines ${ }^{6} 1619$ and encompass the risk factors hypertension, atrial fibrillation and dyslipidaemia. The missed opportunities will be defined as:

1. Untreated high blood pressure: Patients with an average of three blood pressure recordings $\geq 160 \mathrm{~mm} \mathrm{Hg}$ for systolic or $\geq 100 \mathrm{~mm} \mathrm{Hg}$ for diastolic but no antihypertensive medication has been prescribed. 
2. Untreated moderately high blood pressure and at high CVD risk: Patients with an average of three blood pressure recordings $\geq 140 \mathrm{~mm} \mathrm{Hg}$ for systolic or $\geq 90 \mathrm{~mm} \mathrm{Hg}$ for diastolic and have a history of coronary heart disease (CHD), peripheral arterial disease (PAD), chronic kidney disease (CKD), diabetes mellitus and over 40 years or an estimated CVD risk of $\geq 20 \%$ over 10 years but no antihypertensive medication has been prescribed.

3. Atrial fibrillation and at high risk of stroke with no anticoagulant therapy prescribed: Patients with atrial fibrillation and a CHADS2 score $\geq 1$ but no anticoagulant medication prescribed.

4. Patients at high CVD risk or with familial hypercholesterolaemia and no lipid-modifying drug therapy prescribed: High CVD risk will be defined as having a history of CHD, PAD, CKD, diabetes mellitus and over 40 years or an estimated CVD risk of $\geq 20 \%$ over 10 years.

\section{Definition of outcomes and variables Stroke/TIA}

A comprehensive list of stroke and TIA Read codes has been developed to identify the eligible population (see online supplementary appendix 1). A systematic search strategy was conducted to ensure all relevant Read codes were included:

1. Quality Outcomes Framework (QOF) ${ }^{28}$ stroke and TIA Read codes were reviewed for relevance to the study's eligibility criteria. To capture first stroke and TIA, Read codes relating to history of stroke or TIA were removed.

2. To identify additional Read codes not included in QOF, we conducted a hierarchy screening of QOF stroke and TIA Read codes and key word searches using STATA V.12 (College Station, Texas, USA).

3. Literature was searched for additional Read codes and a clinician was consulted.

\section{Missed opportunities variables}

To identify patients with blood pressure $\geq 160 / 100$ or $\geq 140 / 90 \mathrm{~mm} \mathrm{Hg}$, the average of the three most recent systolic and diastolic blood pressure recordings within 3 years prior to the index date will be used. Diagnoses of atrial fibrillation, CHD, CKD, diabetes mellitus and PAD will be identified using QOF Read codes (V.27). ${ }^{28}$ In addition, where present, Read codes indicating history of diagnosis will be used. Similarly, where available, we have identified 'resolved' Read codes (eg, 212H.00 diabetes resolved), which will be used to indicate if the condition resolved before the index date (see online supplementary appendix 2). Familial hypercholesterolaemia is poorly coded in primary care but is associated with total cholesterol of $\geq 9 \mathrm{mmol} / \mathrm{L} .^{29}$ Therefore, in addition to Read codes for familial hypercholesterolaemia, total cholesterol of $\geq 9 \mathrm{mmol} / \mathrm{L}$ (most recent record prior to index date) will be used to indicate familial hypercholesterolaemia.
A missed opportunity will be identified if a patient was eligible for primary prevention drug therapy but was not on relevant treatment at the time of stroke or TIA. To determine if patients were on antihypertensive, anticoagulant or lipid-modifying drug therapies before their stroke or TIA, the most recent prescriptions for these drugs prior to the index date will be extracted. Prescriptions will be identified using drug codes corresponding to relevant BNF chapters (V.67) and relevant Read codes (eg, 66Q..11, anticoagulant monitoring; see online supplementary appendix 3). In primary care, 90 days is the maximum prescribing length for any treatment. Therefore, a missed opportunity will be recorded when patients were eligible for treatment but their most recent prescription was over 90 days from the index date and consequently were not on treatment at the time of stroke or TIA. However, prescribing anticoagulant therapy usually involves referral to an anticoagulant clinic; to account for this, an additional lag period of 30 days will be allowed for anticoagulant prescribing (ie, 120 days from the index date). The length of the lag period was determined though consultation with eight practising GPs.

The Framingham risk equation will be used to calculate CVD risk over 10 years (table 1 ). This risk equation was chosen as it can be incorporated within Vision, the electronic system used by general practices that contribute to the THIN database. In addition, it was the risk score recommended by the guidelines during the majority of the study period ${ }^{16}$ and the equation is freely available. For consistency, the Framingham CVD risk will be calculated at the index date for all eligible patients and in accordance with Vision calculations. ${ }^{30}$ As recommended by the guidelines, the Framingham CVD risk will be adjusted for South Asian ethnicity and family history of premature CHD. ${ }^{16}$ The CHADS2 score will be used to determine stroke risk for patients with atrial

Table 1 Variables required for the Framingham cardiovascular disease risk equation

\begin{tabular}{|c|c|c|}
\hline Variable & Criteria & $\begin{array}{l}\text { Default } \\
\text { value }\end{array}$ \\
\hline Age $^{*}$ & $30-74$ & $\dagger$ \\
\hline Sex & Male/female & $\dagger$ \\
\hline $\begin{array}{l}\text { Systolic blood } \\
\text { pressure }\end{array}$ & $\begin{array}{l}\text { Most recent record prior } \\
\text { to index date }\end{array}$ & $\dagger$ \\
\hline Total cholesterol & $\begin{array}{l}\text { Most recent record prior } \\
\text { to index date }\end{array}$ & 6.0 \\
\hline HDL cholesterol & $\begin{array}{l}\text { Most recent record prior } \\
\text { to index date }\end{array}$ & $\begin{array}{l}\text { Female: } \\
1.4 \\
\text { Male: } 1.15\end{array}$ \\
\hline Smoking & Yes/no & \\
\hline Diabetes mellitus & Yes/no & \\
\hline ECG-LVH & Yes/no & \\
\hline
\end{tabular}


fibrillation (table 2). Similar to the Framingham risk equation, CHADS2 will be used because it can be incorporated within Vision and it will be calculated at the index date in compliance with Vision calculations.

\section{Predictor variables}

Sociodemographic variables will be extracted including Townsend deprivation quintiles, ${ }^{31}$ urban rural scores, ${ }^{31}$ strategic health authority ${ }^{32}$ and ethnicity. Comorbidities will be identified and defined by QOF Read codes (QOF business rules V.27; see online supplementary appendix 2). ${ }^{33}$ To document patients' contact with primary care before their stroke or TIA, the number of consultations in the year prior to the index date and length of registration will be extracted for each patient. In addition, Read codes indicating exceptions for initiating stroke prevention drug therapy will be extracted including white coat hypertension and contraindications to prescribing antihypertensive, anticoagulant or lipidmodifying drugs (eg, 8I3N.00, hypertension treatment refused; see online supplementary appendix 4).

Predictor variables encompassing modifiable and nonmodifiable risk factors for stroke and TIA will be extracted. Non-modifiable risk factors include sex and age at index date, whereas modifiable risk factors relate to lifestyle: body mass index (BMI), smoking and alcohol intake. GPs initiating lifestyle interventions has been reported to delay initiation of antihypertensive drug therapy by up to 12 months ${ }^{21}$ therefore, we have also identified Read codes indicating lifestyle interventions related to smoking, alcohol intake, diet, exercise and weight (see online supplementary appendix 5).

\section{Quality checks, missing data and extreme values}

Absence of a diagnosis code will be taken to indicate the diagnosis is not present. For categorical variables (eg, smoking status), a separate 'missing' category will be created. Extreme values for blood pressure, total and highdensity lipoprotein cholesterol, height and BMI will be identified using the ranges seen in the Health Survey for England statistics as a guide ${ }^{34}$ and excluded. Incidence of stroke and TIA diagnoses will be investigated over time to check for indication of unusual variation which might indicate incorrect clinical coding. If appropriate, a cut-off date will be introduced for quality of reporting.

Table 2 Variables required for the CHADS2 stroke risk equation for patients with atrial fibrillation

\begin{tabular}{lll}
\hline & Variable & Points \\
\hline C & Congestive heart failure & 1 \\
H & Hypertension & 1 \\
A & Age $\geq 75$ years & 1 \\
D & Diabetes mellitus & 1 \\
S2 & Prior stroke or TIA & 2 \\
\hline TIA, transient ischaemic attack.
\end{tabular}

\section{Analysis}

The primary analysis will calculate the proportion of strokes and TIAs with missed opportunities for primary prevention drug therapy. Proportions will be calculated for each missed opportunity: untreated high blood pressure; untreated moderately high blood pressure and high CVD risk; atrial fibrillation and high risk of stroke with no anticoagulant therapy prescribed; high CVD risk or with familial hypercholesterolaemia and no lipid-modifying drug therapy prescribed. In addition, the proportion of patients with two, three or four missed opportunities will be calculated.

Secondary analysis will comprise of multivariable logistic regression modelling to predict the effect of demographic and patient characteristics on missed opportunities. The logistic regression model will be mixed effect and include practice as a random effect. Year of stroke will be included to investigate how missed opportunities have changed over time. We aim to develop a model that fits the data well, is biologically meaningful and can be meaningfully interpreted. To achieve this, explanatory variables will be entered into the logistic regression model which have been prespecified and informed through literature searches and clinical input (table 3). There is compelling evidence from the literature that age and sex are important predictors of non-adherence to guidelines in primary care; ${ }^{21} 3536$ therefore, these variables will be included in the model regardless of statistical contribution. Although the other prespecified variables have been informed through the literature and clinical advice, the evidence is limited; for that reason, a backwards elimination approach will be adopted to inform model selection. Backwards elimination will be used as it is favourable over forwards or stepwise selection. ${ }^{37}$ Traditionally, a $p$ value of $>0.1-0.2$ is used as a criteria to eliminate variables. However, our sample size is expected to be large and consequently we will use a p-to-eliminate value of $>0.05$. Exploratory analysis will be conducted to explore the relationship of the effect of consultation frequency in the year prior to the index date and duration of registration on missed opportunities for stroke and TIA prevention.

\section{DISCUSSION}

This study will quantify the proportion of patients in whom opportunities to prevent strokes and TIAs were missed. In addition, it will identify the risk factors with the highest proportion of untreated patients. The results of the regression model will be important to provide insight into patient characteristics that predict missed prevention opportunities. Dissemination of these findings to GPs will raise awareness of patients who are vulnerable to not being prescribed relevant stroke and TIA prevention pharmacotherapy when eligible. Furthermore, the findings have the potential to change practice and improve patient care.

The strength of this study is that data are available from over 500 general practices and reflect actual practice. 
Table 3 Explanatory variables for logistic regression modelling

\begin{tabular}{ll}
\hline Variable & Categories \\
\hline Age & 5 year age ban \\
Sex & Male, female \\
Townsend deprivation quintiles & $1,2,3,4,5, \mathrm{Mi}$ \\
Urban/rural score & Urban, rural, miss \\
Strategic health authority & East of England, \\
& West, South Ce \\
& Midlands, Yorksh \\
Country & England, North \\
BMI & Healthy, overweig \\
Smoking status & Current smoker, \\
Alcohol intake & High, moderate, \\
Comorbidities: asthma/atrial fibrillation/cancer/CHD/CKD/ & Individually ent \\
COPD/dementia/depression/diabetes mellitus/epilepsy/heart & Number of com \\
failure/hypertension/hypothyroidism/learning disabilities/ & \\
mental health/osteoporosis/palliative care/rheumatoid arthritis & \\
Lifestyle intervention & Yes/no \\
Year of stroke & Year \\
GP practice & Random effect
\end{tabular}

BMI, body mass index; CHD, coronary heart disease; CKD, chronic kidney disease; COPD, chronic obstructive pulmonary disease; GP, general practitioner.

However, the data will be extracted from routinely collected electronic medical records and, therefore, does not capture the decision-making process which occurs during a consultation. For instance, patients' preferences and GPs knowledge of patient's adherence to medication. ${ }^{38}$ Although our study will include comorbidities in the regression model and report Read codes which indicate contraindications for medications, there may be other legitimate reasons for not prescribing stroke prevention drug therapy and patients might decline antihypertensive, anticoagulant or lipid-lowering drug therapy. Inevitably, there will be missing data and errors in data entry; however, this is expected to be a small proportion of the population and we will exclude extreme values and incorporate missing data as a category in the analysis. The use of QOF Read codes to identify comorbidities is likely to result in missing diagnoses that have been recorded using alternative Read codes. However, the use of QOF Read codes provides a consistent method to identify diagnoses and, since being introduced, GPs are incentivised to use QOF Read codes.

In conclusion, this study will offer an insight into whether stroke and TIA risk factors are being managed adequately in UK primary care. Primary prevention of stroke and TIA is important to reduce the burden of these conditions on the NHS and society. If optimal rates of prevention are not being delivered in primary care, dissemination of our findings will be important and further research should be conducted to identify barriers to guideline adherence and intervention(s) to overcome these.

\section{ETHICS AND DISSEMINATION}

Individual studies using THIN data do not require separate ethical approval but must be approved by the independent Scientific Review Committee (SRC). The findings will be disseminated through publication in a peer-reviewed journal and presented at national and international conferences.

Contributors GMM led the design of the study as doctoral research supervised by TM, MC and MGF. GMM drafted the manuscript. TM, MC and MGF provided feedback on the manuscript and all authors approved the final version.

Funding This work is supported by the National Institute for Health Research (NIHR) School for Primary Care Research (SPCR).

Competing interests None.

Ethics approval This study was approved by the SRC on 31 May 2013 (reference number: 13-023)

Provenance and peer review Not commissioned; externally peer reviewed.

Open Access This is an Open Access article distributed in accordance with the Creative Commons Attribution Non Commercial (CC BY-NC 4.0) license, which permits others to distribute, remix, adapt, build upon this work noncommercially, and license their derivative works on different terms, provided the original work is properly cited and the use is non-commercial. See: http:// creativecommons.org/licenses/by-nc/4.0/

\section{REFERENCES}

1. Scarborough $\mathrm{P}$, Peto V, Bhatnagar $\mathrm{P}$, et al. Stroke statistics. London: British Heart Foundation and Stroke Association, 2009.

2. Townsend N, Wickramasinghe K, Bhatnagar P, et al. Coronary heart disease statistics 2012 edition. London: British Heart Foundation, 2012.

3. The Lancet Editorial. Stroke-prevention is better than cure. Lancet 2007;369:247

4. Lewington S, Clarke R, Qizilbash N, et al. Age-specific relevance of usual blood pressure to vascular mortality: a meta-analysis of individual data for one million adults in 61 prospective studies. Lancet 2002;360:1903-13.

5. MacMahon S, Peto R, Cutler J, et al. Blood pressure, stroke, and coronary heart disease. Part 1, prolonged differences in blood pressure: prospective observational studies corrected for the regression dilution bias. Lancet 1990;335:765-74.

6. National Collaborating Centre for Chronic Conditions. Atrial fibrillation: national clinical guideline for management in primary and secondary care. London: Royal College of Physicians, 2006. 
7. Zhang $\mathrm{X}$, Patel $\mathrm{A}$, Horibe $\mathrm{H}$, et al. Cholesterol, coronary heart disease, and stroke in the Asia Pacific region. Int J Epidemiol 2003;32:563-72.

8. The Prospective Studies Collaboration. Cholesterol, diastolic blood pressure, and stroke: 13,000 strokes in 450,000 people in 45 prospective cohorts. Prospective studies collaboration. Lancet 1995;346:1647-53.

9. Amarenco P, Labreuche J, Lavallee $\mathrm{P}$, et al. Statins in stroke prevention and carotid atherosclerosis: systematic review and up-to-date meta-analysis. Stroke 2004;35:2902-9.

10. Goldstein LB, Bushnell CD, Adams RJ, et al. Guidelines for the primary prevention of stroke: a guideline for healthcare professionals from the American Heart Association/American Stroke Association. Stroke 2011;42:517-84.

11. Brown CD, Higgins M, Donato KA, et al. Body mass index and the prevalence of hypertension and dyslipidemia. Obes Res 2000;8:605-19.

12. Hollander M, Koudstaal PJ, Bots ML, et al. Incidence, risk, and case fatality of first ever stroke in the elderly population. The Rotterdam Study. J Neurol Neurosurg Psychiatry 2003;74:317-21.

13. Appelros $P$, Stegmayr B, Terent A. Sex differences in stroke epidemiology: a systematic review. Stroke 2009;40:1082-90.

14. Tillin T, Hughes A, Mayet J, et al. The relationship between metabolic risk factors and incident cardiovascular disease in Europeans, South Asians, and African Caribbeans: SABRE (Southall and Brent Revisited)—a prospective population-based study. J Am Coll Cardiol 2013;61:1777-86.

15. British Cardiac Society, British Hypertension Society, Diabetes UK, HEART UK, Primary Care Cardiovascular Society, The Stroke Association. JBS 2: Joint British Societies' guidelines on prevention of cardiovascular disease in clinical practice. Heart 2005;91 (Suppl V):1-52.

16. National Institute for Health and Clinical Excellence. Lipid modification: cardiovascular risk assessment and the modification of blood lipids for the primary and secondary prevention of cardiovascular disease. NICE clinical guideline 67. National Collaborating Centre for Primary Care, 2008.

17. The Stroke Risk in Atrial Fibrillation Working Group. Independent predictors of stroke in patients with atrial fibrillation: a systematic review. Neurology 2007;69:549-54.

18. Law MR, Wald NJ, Rudnicka AR. Quantifying effect of statins on low density lipoprotein cholesterol, ischaemic heart disease, and stroke: systematic review and meta-analysis. BMJ 2003;326:1423-7.

19. National Institute for Health and Clinical Excellence. Hypertension: the clinical management of primary hypertension in adults. Clinical Guideline 127. National Clinical Guideline Centre, 2011.

20. Guptha SH, Shibu P, Owusu-Agyei P. Stroke prevention: missed opportunities. Lancet 369:904-5.
21. Midlov P, Ekesbo R, Johansson L, et al. Barriers to adherence to hypertension guidelines among GPs in southern Sweden: a survey. Scand J Prim Health Care 2008;26:154-9.

22. Steinman MA, Fischer MA, Shlipak MG, et al. Clinician awareness of adherence to hypertension guidelines. Am J Med 2004;117:747-54.

23. CSD Medical Research UK. CSD Medical Research UK [cited September 2014]. http://csdmruk.cegedim.com/index.htm

24. British National Formulary (BNF). British National Formulary (BNF) 67 2014. http://www.bnf.org/bnf/index.htm

25. Health and Social Care Information Centre. Read Codes 2014 [cited September 2014]. http://systems.hscic.gov.uk/data/uktc/ readcodes

26. CDS Health Research. The Health Improvement Network Ethics [cited September 2014]. http://www.thin-uk.com/mrec.htm

27. Maguire A, Blak B, Thompson M. The importance of defining periods of complete mortality reporting for research using automated data from primary care. Pharmacoepidemiol Drug Saf 2009;18:76-83.

28. Health and Social Care Information Centre. Quality and Outcomes Framework (QOF) 2014 [cited September 2014]. http://www.hscic. gov.uk/qof

29. Koivisto PV, Koivisto UM, Miettinen TA, et al. Diagnosis of heterozygous familial hypercholesterolemia. DNA analysis complements clinical examination and analysis of serum lipid levels. Arterioscler Thromb Vasc Biol 1992;12:584-92.

30. In Practice Systems Ltd. CVD/Stroke Risk Calculators within Vision 2012 [cited September 2014]. http://www.inps.co.uk/my-vision/ user-guides-downloads/user-guides/releases/vision-releases

31. CSD Medical Research UK. THIN Data Guide for Researchers. 2013; Vol 2.6.

32. Public Health England. Strategic Health Authorities in England 2014 [cited September 2014]. http://www.swpho.nhs.uk/default.aspx? $\mathrm{RID}=27853$

33. Primary Care Commissioning. QOF business rules v27 2013 [cited September 2014]. http://www.pcc-cic.org.uk/article/ qof-business-rules-v27

34. Health Survey for England. Health Survey for England (HSE)-2012 adult trend tables 2012 [cited September 2014]. http://www.hscic. gov.uk/pubs/hse10trends

35. Ramsay SE, Whincup PH, Wannamethee SG, et al. Missed opportunities for secondary prevention of cerebrovascular disease in elderly British men from 1999 to 2005 : a population-based study. $J$ Public Health 2007;29:251-7.

36. McKinlay JB, Link CL, Freund KM, et al. Sources of variation in physician adherence with clinical guidelines: results from a factorial experiment. J Gen Intern Med 2007;22:289-6.

37. Mantel N. Why stepdown procedures in variable selection. Technometrics 1970;12:621-5.

38. Cabana MD, Rand CS, Powe NR, et al. Why don't physicians follow clinical practice guidelines? A framework for improvement. JAMA $1999 ; 282: 1458-65$ 